\title{
Editorial
}

\section{The Health Reform Moment: Peril and Possibility in the Obama Era}

\author{
Daniel Redwood, D.C.
}

$\mathbf{F}$ or the first time in 15 years, a major national health reform initiative is moving forward in the United States. Those of us who recall the events of 1993-1994, when the Clinton administration failed to pass its version of coverage for all, know that numerous pitfalls lie ahead with the potential to undermine the best-laid plans.

But for those of us who have seen the widespread and needless suffering caused by the dominant role of money in American health care, President Barack Obama's clear commitment to change gives much cause for optimism. Currently, tens of millions of uninsured Americans lack adequate access to quality health services and uncounted millions more delay or decline needed care for financial reasons. The men and women leading the health reform process, particularly at the interface of the executive and legislative branches of the federal government, appear to have learned from past failures. Also of considerable significance, the new president is a consensus builder with roots as a community organizer and is now applying his unprecedented grassroots-plus-online campaign model to the process of governance.

Access to care and related budgetary issues tend to dominate the debate, but they comprise only one part of the equation. In the long run, financial issues may prove less important than questions about which services are being delivered to patients with newly increased access, as well as to those who already have access to health care but for whom it has failed to deliver health and wellness. Unless there are major changes in the health priorities of the nation, there will be no sustainable health care solutions. The stakes are extremely high.

This commentary focuses on four areas: universal coverage; prevention and health promotion; chiropractic (I am a chiropractic educator who was in private practice for 26 years); and complementary and alternative medicine (CAM).

\section{A Right, Not a Privilege}

For this journal's international readers, the fact that the United States remains the only industrialized nation without universal health coverage must seem truly bizarre. Health care costs are the leading cause of personal bankruptcies in the United States, and fear of financial ruin dra- matically compounds the stresses inherent in major illness. Appreciation of health care as a right rather than a privilege now appears to have reached critical mass in the United States, several decades later than in most other nations. This alone would probably not prove sufficient as a driver of systemic change; the other new factor on the health reform landscape is that the competitive disadvantage for large American businesses, many of which spend vast amounts on their employees' health care, has passed a key tipping point in recent years. This had not yet occurred at the time of the failed Clinton health initiative in the 1990s.

\section{The Primacy of Prevention}

As the debate on health care reform heats up, one area of unanimous agreement stands out-people on all sides agree that it is far better to prevent illness than to treat disease after it takes root. The healing arts originated in response to the fundamental human need to relieve pain and suffering. Over time, the most insightful physicians and healers in all cultures developed the principles and practices of disease prevention and health promotion that we now increasingly recognize as the highest expression of the healing arts.

But while there is widespread agreement about the primacy of prevention in principle, divergent views arise as soon as discussion shifts to specific priorities, goals, and methods of implementation. When the Obama-Biden Transition Health Policy Team offered three examples of preventive services in its guide for participants in Community Health Care Discussions (my college hosted one of the 8500-plus meetings held in December 2008), the team skewed sharply toward a medicalized view of prevention, with early disease detection accorded the highest priority. The three examples were mammography, flu shots and cholesterol screenings.

Detecting disease early is, of course, far better than detecting it late. But the true "holy grail" of prevention is found not in detecting disease but in undertaking the lifestyle changes-whole foods diet, regular exercise, stress management, and social support-that keep us well. There are clear indications that the Obama team recognizes the importance of these lifestyle changes. The key question is: Will this recog- 
nition lead to a true paradigm shift, with large-scale increases in lifestyle-based primary prevention efforts backed by genuine budgetary muscle or will it amount to little more than lip service? In the coming months, generating support for broad-scale primary prevention through lifestyle changes must be among Americans' highest priorities.

It is worth noting that if every primary care medical physician in the nation delivered all preventive health services recommended for every patient by the U.S. Preventive Services Task Force, these physicians would have no time for anything else. ${ }^{1}$ There would be no time for other patient care, no record keeping, no time for continuing education, and no time for creative thinking. Therefore, simple mathematics dictates that either preventive services recommendations must be drastically scaled back (precisely the opposite of what policy analysts from left, right, and center are proposing) or other delivery channels must be developed. Any meaningful prevention and health promotion plan must mobilize the efforts of other health providers (nurses, physician's assistants, chiropractors, naturopaths, acupuncturists, and others) for these preventive services, consistent with these practitioners' training and licensure. Like medical physicians, however, these other providers have additional responsibilities. Enlisting their help is necessary but far from sufficient.

A new prevention and health promotion infrastructure must be developed as well, along the lines of the Samueli Institute's (Alexandria, VA) superbly crafted Wellness Initiative for the Nation (WIN), which proposes implementation of new policies to "establish standards in comprehensive lifestyle and integrated health care approaches, and train individuals with qualifications to focus full-time on prevention." A new cadre of Health and Wellness Coaches is envisioned to fill the gaping holes in our current prevention and health promotion infrastructure. Also included in the WIN model are "specialist certification for health professionals in prevention, health and wellness delivery in specific settings and populations - for example, schools, worksites, the military, health care settings, and long-term care facilities," and creating "a Health Corps to provide an army of young and older people that would learn and model wellness behavior and support delivery of wellness education and training by the coaches." The current draft of WIN-at www.siib.orgshould be read by everyone seeking to understand the transformative potential of the health reform moment.

\section{Chiropractic: Time for Full Inclusion}

As a profession that over the past generation has made great strides into the American health care mainstreamwith widespread utilization and patient satisfaction; a strong research base; inclusion in most private insurance plans, worker's compensation insurance, Medicare, military, and veterans health care; and full recognition in Olympic and sports medicine-chiropractic now has the hallmarks of an essential health service.

Whether it is specifically recognized as essential in the core benefits package of the emerging health reform plan may prove to be a bellwether (along with lifestyle-based prevention) as to the extent to which genuine, paradigm-shifting change is embodied in the Obama program. Chiropractors (D.C.s) and their 22 million patients in the United States were quite heartened when Obama sent three separate letters of support to the American Chiropractic Association (ACA) during the presidential campaign. Most encouraging was the fact that Obama, the eventual winner, was the only presidential candidate in either party to specifically respond to the ACA's detailed candidate questionnaire.

Aside from inclusion in a core benefit package, arguably the most critical goal for chiropractors (and other non-M.D. practitioners), is to codify in federal law a policy of nondiscrimination among types of providers, for both coverage and reimbursement. In other words, if spinal manipulation (or massage, physical rehabilitation, nutritional counseling, mindfulness meditation instruction, or any other procedure) is covered when performed by a medical or osteopathic physician, then it must always be covered at the same rate of reimbursement for any health practitioner licensed to provide it. Freedom of choice among providers and a level playing field on coverage and reimbursement are ideas whose time has hopefully arrived.

Recognition of chiropractors as an essential part of the U.S. health care system also dovetails exceedingly well with the expanded lifestyle-based prevention efforts that must form the leading edge of health policy in the coming era. Chiropractors have always encouraged patients to take an active role in restoring and maintaining health, with particular emphasis on doctor-guided self-care through exercise and nutrition, ${ }^{2}$ and new profession-wide initiatives reflect a deepened commitment to prevention and health promotion across the wide range of parameters elaborated by the U.S. Preventive Services Task Force. All students graduating from chiropractic colleges after January 2007 have been required to demonstrate knowledge of evidence-based prevention approaches and mastery of methods for applying these approaches in the clinical setting. This wellness mandate by the Council on Chiropractic Education, the chiropractic accrediting agency, grants individual institutions leeway to craft their own prevention training programs, but all institutions are audited for compliance with the key markers spelled out in the mandate. Thus, all future chiropractic graduates will be required to demonstrate evidence-based wellness assessment and intervention skills, which presumably will carry over into their careers as practicing chiropractors.

One further goal for chiropractors in the health reform process is broadened inclusion of D.C.s on federal, state, and private sector health policy committees of all types. Such inclusion has expanded slowly over the past decade or two. When implemented, it accrues to the benefit of the health care system as a whole, and the millions of patients it serves, by diversifying the mix of ideas and perspectives brought to the table. The knowledge infrastructure that now exists within the chiropractic profession (including many dual-degreed practitioners) justifies the inclusion of qualified chiropractors on health care committees across the board, not just those with a musculoskeletal focus.

\section{The Role of CAM}

Because CAM encompasses a wide range of procedures, disciplines, and professions, generalizations about the inclusion of CAM in health care reform are far more difficult to frame than those for prevention or chiropractic. Single therapies such as St. John's wort (Hypericum perforatum) for depression or Echinacea (Echinacea spp.) for colds do not comfortably cohabit the same category as entire systems such as Chinese medicine or naturopathic medicine. But a quick 
perusal of the areas under the aegis of the National Center for Complementary and Alternative Medicine at the U.S. National Institutes of Health $(\mathrm{NIH})$ indicates that such odd groupings do indeed reflect the current definition of CAM.

It is possible, nonetheless, to develop consensus principles, strategies, and desired outcomes for the role of CAM in health reform. The final report of the White House Commission on Complementary and Alternative Medicine Policy, chaired by CAM pioneer James S. Gordon, M.D., provides the best available roadmap. Anyone arguing for the inclusion of CAM (or any of its constituent parts) in a reformed health care system would do well to consult this document (www.whccamp.hhs.gov) for its thorough analysis and policy prescriptions.

CAM's contributions to health reform should grow organically from its areas of greatest strength. Foremost among these is the principled and pragmatic insistence that health be understood holistically, as a summation of various lifeaffirming inputs and not the mere absence of symptoms. The key corollary - still not sufficiently accepted by conventional medicine-is that, while single-intervention "silver bullet" therapies may dramatically eliminate symptoms (sometimes in life-saving ways), these therapies alone are not the road to long-term, sustainable health and wellness.

Thus, perhaps the most important policy change relevant to CAM would be to shift both CAM and conventional health research budgets away from the longstanding emphasis on single intervention therapeutics and toward multifactorial integrative and whole-systems approaches. (The Ornish lifestyle program that proved heart disease could be reversed $^{3,4}$ through a combination of diet, exercise, stress management, and social support provides the best model for this approach.) Whether for heart disease, low-back pain, prostate cancer, or any other condition, NIH policies that funnel virtually all health research funds to single-agent (usually pharmaceutical) studies have led us into a cul-de-sac of historic proportions. This research strategy may have been effective for combating many infectious diseases, but it has proven far less applicable to the chronic diseases-cancer, heart disease, diabetes, obesity, arthritis, osteoporosis, and others - that are the bane of the developed world. CAM can play a helpful role in finding a way forward.

One other issue of great importance for both CAM and the rest of the health care system is that there needs to be a national policy commitment to train all health practitionersas students and later through continuing education-to deliver basic information on a whole-foods diet, exercise, and stress management. There is no lack of scientific evidence for these approaches; what is lacking is a deep appreciation of their importance and the will to teach these to the patients who so desperately need them.

Moreover, all practitioners of all types must become conversant with the full range of health care approaches (both conventional and CAM) available in the U.S. health care system. This does not mean that medical internists must themselves deliver spinal manipulation, acupuncture, or massage treatments; it means that such internists need to know what these therapies involve and where evidence supports their appropriate use. When those circumstances arise, referrals should be made. This is a two-way street; CAM practitioners must also be well-informed about the biomedical options available to patients in order to make appropriate referrals, and must not hesitate to do so. It must never be considered acceptable for either conventional or CAM practitioners to be ignorant of what other health care approaches have to offer. Wide-ranging knowledge and mutual respect must be our watchwords.

Failure to inform patients of the available options is a form of negligence. ${ }^{5}$ This is particularly egregious when a negative outcome results from an invasive therapy and the patient was not informed of less-invasive, evidence-based alternatives. The overarching goal for all practitioners must be collaboration and cooperation for the benefit of our patients.

\section{Status Quo with Continuing Decline or a Historic Step Forward?}

The current health reform initiative is a high-impact event for all concerned. Failure by Congress and the Obama administration to enact universal coverage now would be likely to doom such efforts for a generation. Failure to properly fund and prioritize lifestyle-based prevention methods would more deeply entrench a status quo that has already proven woefully inadequate at serving society's health needs. Failure to institute a level playing field among the professions would be tantamount to an endorsement of ongoing injustice. Excluding established professions that provide essential services from a national core benefit plan would mark a major step backward. Make no mistake-a very real possibility of disaster on many levels lurks in the shadows.

But President Obama's health reform effort also offers the best chance we've seen in our lifetimes to take a truly large and historic step forward, with coverage for all, nondiscrimination among providers, a commitment to research the integrative approaches that may hold the best hope for dealing with our era's epidemic levels of chronic disease, and an all-hands-on-deck national program for lifestyle-based prevention and health promotion. The possibilities are breathtaking. This is a time to dream big dreams.

Everything is on the table. The potential risks and rewards could not be greater. Make your views known. Be part of the solution. What happens next is up to all of us.

\section{References}

1. Yarnall KS, Pollak KI, Ostbye T, et al. Primary care: Is there enough time for prevention? Am J Public Health 2003;93:635-641.

2. Redwood D, Globe G. Prevention and health promotion by chiropractors. Am J Lifestyle Med 2008;2:537-545.

3. Ornish D, Brown SE, Scherwitz LW, et al. Can lifestyle changes reverse coronary heart disease? The Lifestyle Heart Trial. Lancet 1990;336:129-133.

4. Ornish D, Scherwitz LW, Billings JH, et al. Intensive lifestyle changes for reversal of coronary heart disease. JAMA 1998;280:2001-2007.

5. Cohen MH, Ruggie M, Micozzi MS. The Practice of Integrative Medicine: A Legal and Operational Guide. New York: Springer; 2006.

Address reprint requests to: Daniel Redwood, D.C. Cleveland Chiropractic College-Kansas City 10850 Lowell Avenue Overland Park, KS 66213

E-mail: dan.redwood@cleveland.edu 